\title{
The double degenerate LP 400-22 revisited
}

\author{
S. Vennes ${ }^{1, \star}$, A. Kawka $^{1}$, T. R. Vaccaro ${ }^{2, \star}$, and N. M. Silvestri ${ }^{3}$ \\ 1 Astronomický ústav AV ČR, Fričova 298, 25165 Ondřejov, Czech Republic \\ e-mail: vennes, kawka@sunstel.asu.cas.cz \\ 2 Department of Physics \& Astronomy, Francis Marion University, Box 100547, Florence, SC 29501, USA \\ e-mail: tvaccaro@fmarion.edu \\ 3 Department of Astronomy, University of Washington, Box 351580, Seattle, WA 98195, USA \\ e-mail: nms@astro.washington.edu
}

Received 22 July 2009 / Accepted 17 September 2009

\section{ABSTRACT}

\begin{abstract}
We re-examine the properties of the high-velocity and extremely low-mass white dwarf LP 400-22 and its close companion. Based on an extended observation timeline, we determined a binary period of $P=1.01016 \mathrm{~d}$, somewhat longer than the previously published period, and a mass function $f\left(M_{2}\right)=0.180 M_{\odot}$, implying a mass for the companion $M_{2}>0.41 M_{\odot}$. We also re-appraised the mass and cooling age of the white dwarf using low-metallicity $(Z=0.001)$ evolutionary models appropriate for an old halo member, $M_{1}=0.19 M_{\odot}$, and $t_{\text {cool }} \approx 1.8 \mathrm{Gyr}$, and we infer a mass of $0.85-1.0 M_{\odot}$ for the progenitor of the white dwarf. We discuss the likely origin of this system.
\end{abstract}

Key words. stars: binaries: close - stars: individual: LP 400-22 - white dwarfs

\section{Introduction}

LP 400-22 (NLTT 54331) is an extremely low-mass (ELM) white dwarf identified by Kawka et al. (2006). This object is also remarkable for its peculiar kinematics characterised by a high tangential velocity $v_{\tan } \approx 400 \mathrm{~km} \mathrm{~s}^{-1}$ (Kawka et al. 2006) and for its membership in a $\sim 1$ day binary (Kilic et al. 2009). The companion still has to be identified but it is most likely another white dwarf or a neutron star. Evolutionary scenarios (see Willems \& Kolb 2002; Nelemans et al. 2001) that produce low-mass white dwarfs typically involve a common-envelope (CE) phase responsible for orbital shrinkage, while the most massive component climbs toward the giant branch or, alternatively, while it reaches the asymptotic giant branch. The most massive component eventually collapses to form a neutron star or a white dwarf, and, depending on the resulting orbital period and on the secondary mass, the system may experience episodes of Roche-lobe overflow (RLOF), while the secondary is still on the main-sequence or, later, while it climbs the giant branch. Either path leads to the formation of a low-mass helium white dwarf, with a total systemic age characteristic of the old disk or halo population for systems with a low initial secondary mass, or significantly lower ages for systems with higher secondary masses.

Knowledge of the total age of the system is critical in helping estimate the likely mass and metallicity of the white dwarf progenitor, hence in justifying a particular choice of evolutionary models. In this regard, a kinematical study of the remnant stars is useful in estimating the epoch of formation (Chiba \& Beers 2000).

A few ELM white dwarfs $\left(M<0.2 M_{\odot}\right)$ have been identified as companions to pulsars (van Kerkwijk et al. 1996;

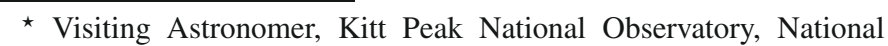
Optical Astronomy Observatory, which is operated by the Association of Universities for Research in Astronomy (AURA) under cooperative agreement with the National Science Foundation.
Bassa et al. 2006). In addition, the white dwarf SDSS J0917+46, which was discovered as part of a search for hypervelocity Btype stars (Kilic et al. 2007a), is also in a $7.5936 \pm 0.0024 \mathrm{~h}$ binary (Kilic et al. 2007b) with a companion still to be classified $^{1}$.

In an effort to improve our understanding of the binary LP 400-22, we obtained two series of intermediate resolution spectra (Sect. 2), which we use to refine the white dwarf atmospheric parameters (Sect. 3.1). Based on new and published radial velocity measurements, we constrain the binary ephemeris, and revise the kinematics of the system, as well as the mass-function for the unseen companion (Sect. 3.2). We also re-evaluate the parameters of the white dwarf based on low-metallicity evolutionary models appropriate for an old halo member (Sect. 3.3). Finally, we present our conclusions (Sect. 4).

\section{Spectroscopy}

Table 1 shows the log of observations. LP 400-22 was observed on 2006 September 30 using the dual imaging spectrograph (DIS) attached to the $3.5 \mathrm{~m}$ telescope at the Apache Point Observatory (APO). We obtained six exposures of 30 min each. We used the 830.8 lines $\mathrm{mm}^{-1}$ grating on the red side to obtain a spectral range of 6440 to $8150 \AA$ with a dispersion of $0.84 \AA$ pixel $^{-1}$, and the 1200 lines $\mathrm{mm}^{-1}$ grating on the blue side to obtain a spectral range of 3830 to $5030 \AA$ with a dispersion of $0.62 \AA \operatorname{pixel}^{-1}$. The slit width was set at $1.5^{\prime \prime}$ resulting in a resolution of $2.1 \AA$ in the red and $1.7 \AA$ in the blue. We concurrently obtained a series of HeNeAr comparison arcs. We corrected the wavelength scale for each science exposure using

\footnotetext{
1 Proceeding by elimination, Agüeros et al. (2009) argue in favour of a white dwarf companion.
} 


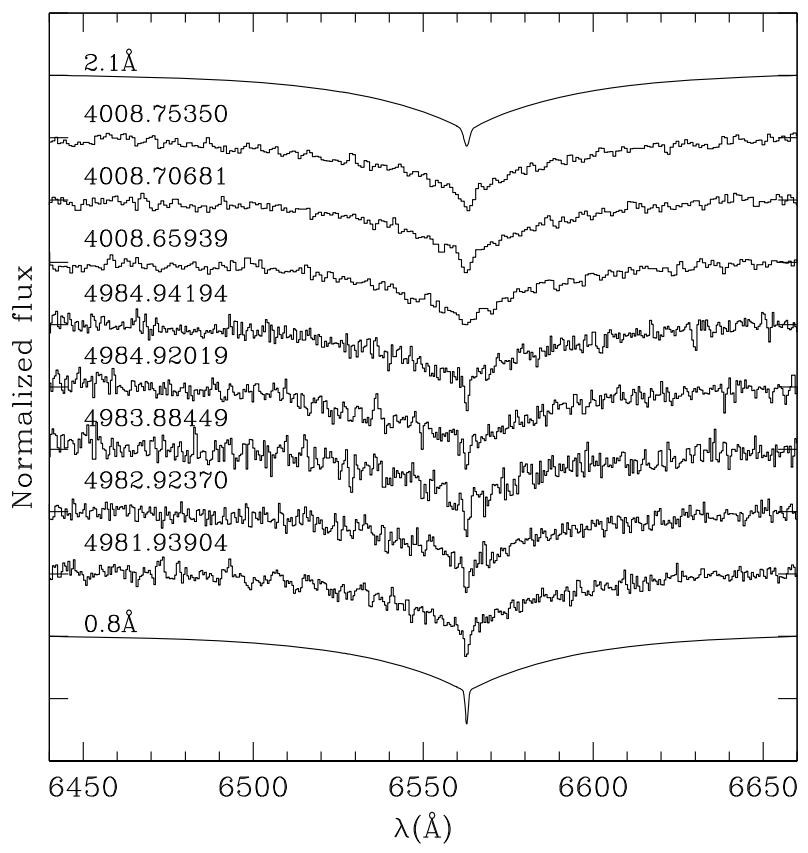

Fig. 1. APO and KPNO spectra centred on $\mathrm{H} \alpha$ (labelled with HJD) compared to synthetic spectra (labelled with spectral resolution) showing a narrow photospheric absorption core.

Table 1. Observation log.

\begin{tabular}{|c|c|c|c|c|}
\hline Observatory & Start UT date & Time & Object & $t_{\exp }(\mathrm{s})$ \\
\hline \multirow[t]{10}{*}{ APO } & 2006 Sep. 30 & $03: 11: 01$ & LP 400-22 & 1800 \\
\hline & & $03: 42: 22$ & $\mathrm{HeNeAr}$ & 90 \\
\hline & & $03: 45: 05$ & LP 400-22 & 1800 \\
\hline & & $04: 16: 20$ & $\mathrm{HeNeAr}$ & 180 \\
\hline & & 04:20:44 & LP 400-22 & 1800 \\
\hline & & 04:51:57 & LP 400-22 & 1800 \\
\hline & & 05:23:09 & $\mathrm{HeNeAr}$ & 180 \\
\hline & & 05:28:07 & LP 400-22 & 1800 \\
\hline & & 05:59:00 & LP 400-22 & 1800 \\
\hline & & $06: 30: 12$ & $\mathrm{HeNeAr}$ & 180 \\
\hline \multirow[t]{9}{*}{ KPNO } & 2009 May 30 & $10: 18: 41$ & LP 400-22 & 1800 \\
\hline & & $10: 50: 38$ & $\mathrm{HeNeAr}$ & 30 \\
\hline & 2009 May 31 & 09:56:28 & LP 400-22 & 1800 \\
\hline & & $10: 28: 23$ & $\mathrm{HeNeAr}$ & 30 \\
\hline & 2009 May 01 & 08:59:53 & LP 400-22 & 1800 \\
\hline & & 09:32:08 & $\mathrm{HeNeAr}$ & 30 \\
\hline & 2009 May 02 & 09:51:10 & LP 400-22 & 1800 \\
\hline & & $10: 22: 29$ & LP 400-22 & 1800 \\
\hline & & $10: 55: 07$ & $\mathrm{HeNeAr}$ & 30 \\
\hline
\end{tabular}

the $\mathrm{HgI} \lambda 4358.335$ sky line on the blue side and the OH $\lambda 6533.05$ sky line on the red side.

Next, we observed LP 400-22 from 2009 May 29 to June 2 using the Ritchey-Chretien spectrograph attached to the $4 \mathrm{~m}$ telescope at Kitt Peak National Observatory (KPNO). We used the KPC-24 grating (860 lines $\mathrm{mm}^{-1}$ ) in the second order resulting in a dispersion of $0.51 \AA$ pixel $^{-1}$ and a spectral range from 6230 to $6860 \AA$. The slit width was set to $1.5^{\prime \prime}$, resulting in a resolution of $\approx 0.8 \AA$ at $\mathrm{H} \alpha$ and degrading to $\approx 1.5 \AA$ on the fringes. We also secured a concurrent series of HeNeAr comparison arcs. To ascertain the stability of the wavelength scale, we also observed the radial velocity standard HD 112299 each night. We measured an average velocity and dispersion $v_{\text {helio }}=4.6 \pm 1.5 \mathrm{~km} \mathrm{~s}^{-1}$ close to published values ( $v_{\text {helio }}=3.9 \pm 0.6 \mathrm{~km} \mathrm{~s}^{-1}$, Mazeh et al. 1996). Moreover, the radial velocity measurements of the sky line OH $\lambda 6533.05$ demonstrate the stability of the wavelength scale with a standard deviation of only $0.7 \mathrm{~km} \mathrm{~s}^{-1}$.

The spectra were flux-calibrated using the spectrophotometric standards Feige 110, Feige 34, and HZ 44. Finally, all spectra were reduced using standard IRAF procedures. Figure 1 shows the spectra near $\mathrm{H} \alpha$. To improve the signal-tonoise ratio of the APO observations we co-added the spectra by pairs. The sharp $\mathrm{H} \alpha$ absorption core proved to be a precise velocity marker in both science, particularly those obtained at KPNO, and velocity standard exposures.

Table 2 shows measured radial velocities and mid-exposure times. The velocities at KPNO were obtained by fitting Voigt profiles to the narrow $\mathrm{H} \alpha$ absorption core using the IRAF 'splot' routine. An average error was estimated by taking successive measurements varying the pseudo-continuum location. We measured the velocities at APO by calculating the average and standard deviation of five independent measurements obtained by fitting Voigt profiles to (1) the $\mathrm{H} \alpha$ line core and (2) the $\mathrm{H} \gamma$ line core, and by (3) cross-correlating the spectra with a template between 6540 and $6580 \AA$, (4) between 4300 and $4400 \AA$, and (5) between 4325 and $4355 \AA$. The red and blue templates were obtained by summing all available spectra of LP 400-22.

\section{Analysis}

\subsection{White dwarf parameters}

We fitted the upper Balmer line profiles obtained at APO using a grid of model atmospheres in local thermodynamic equilibrium (LTE). The model atmospheres include the effect of convection although for this particular model less than $\sim 3 \%$ of the flux is carried through convection at $\tau_{\mathrm{R}} \approx 1$. More importantly, the models include the effect of hydrogen line-blanketing that induces cooling of low optical depth layers, and stimulates the emergence of a narrow Balmer absorption core clearly seen at $\mathrm{H} \alpha$. Residuals to Balmer line fits are often observed, particularly in line cores, which may stem from non-LTE effects in hotter atmospheres, or possibly missing opacities. The new parameters are consistent with our previous analysis and that of Kilic et al. (2009). Table 3 summarises the observed properties of the white dwarf in the binary LP 400-22. We adopted an average of published values of the effective temperature and surface gravity.

\subsection{Binary parameters}

Figure 2 shows the periodograms and best fit radial velocity curve. We fitted the radial velocity measurements, weighted according to Table 2, with sinusoidal functions while varying the period $P$, the mean and semi-amplitude velocities $\gamma$ and $K$, and the initial epoch $T_{0}$. The corresponding $\chi^{2}$ values were then normalised to the best-fitting $\chi^{2}$ and the significance levels drawn at 66,90 , and $99 \%$.

We analysed two separate data sets. The first, using the measurements of Kilic et al. (2009) alone, imply a period of $P=0.9878$ and a minimum $\chi^{2}=2.5$, but with 4 other periods that cannot be excluded with $99 \%$ confidence. Combining our data with their data, we selected a single period with $99 \%$ confidence and a minimum $\chi^{2}=2.2$. A solution excluding the less precise data from APO delivers an identical, although less accurate, period determination.

Based on the joint data set we determined that

$P=1.01016 \pm 0.00005 \mathrm{~d}$,

$T_{0}=2454008.514 \pm 0.025$, 

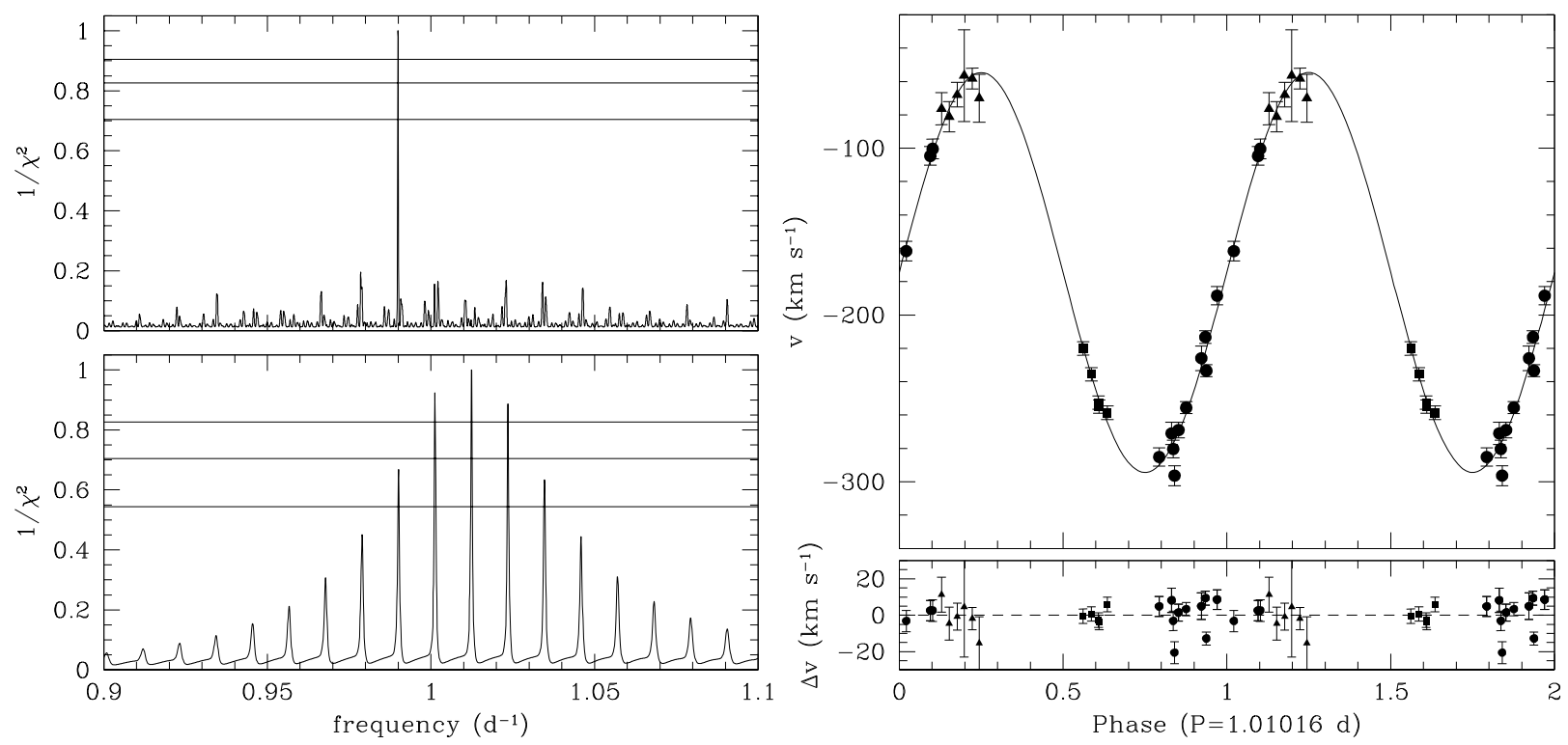

Fig. 2. (Left) Periodogram of published and new radial velocities of the binary LP 400-22 for (top) the combined data set (APO, KPNO and Kilic et al. 2009), and (bottom) for the data set of Kilic et al. (2009) alone. The analysis shows that the correct period is $P=1.01016 \mathrm{~d}$. (Right) Measured velocities and residuals folded on our ephemeris: the triangles are from APO, the squares from KPNO, and the circles from Kilic et al. (2009).

Table 2. Radial velocities.

\begin{tabular}{llr}
\hline \hline Observatory & $\mathrm{HJD}^{a}(2450000+)$ & $v_{\text {helio }}\left(\mathrm{km} \mathrm{s}^{-1}\right)$ \\
\hline APO & 4008.64757 & $-76.3 \pm 9.6$ \\
& 4008.67122 & $-81.1 \pm 9.0$ \\
& 4008.69597 & $-67.9 \pm 7.4$ \\
& 4008.71765 & $-56.4 \pm 27.5$ \\
& 4008.74278 & $-58.2 \pm 6.2$ \\
KPNO & 4008.76422 & $-70.0 \pm 14.4$ \\
& 4981.93904 & $-258.6 \pm 4.0$ \\
& 4982.92370 & $-254.9 \pm 4.0$ \\
& 4983.88449 & $-220.1 \pm 4.0$ \\
& 4984.92019 & $-235.6 \pm 4.0$ \\
& 4984.94194 & $-252.5 \pm 4.0$ \\
\hline
\end{tabular}

${ }^{a}$ At mid-exposure.

where $T_{0}$ is the initial epoch of superior conjunction of the unseen companion $(\Phi=0)$. We determined residuals of 4,8 , and $8 \mathrm{~km} \mathrm{~s}^{-1}$ for the measurements from KPNO, APO, and Kilic et al. (2009), respectively. We subtracted the effect of an estimated gravitational redshift of $\gamma_{\mathrm{g}}=\sqrt{G M g} / c \approx 2.5 \mathrm{~km} \mathrm{~s}^{-1}$ from the apparent systemic velocity $\gamma=-174.5 \pm 1.3 \mathrm{~km} \mathrm{~s}^{-1}$ and determined the systemic velocity:

$\gamma_{\mathrm{sys}}=-177.0 \pm 1.3 \mathrm{~km} \mathrm{~s}^{-1}$.

The projected velocity semi-amplitude of the white dwarf is

$K=119.9 \pm 2.0 \mathrm{~km} \mathrm{~s}^{-1}$,

corresponding to a mass function for the unseen companion of

$f=0.180 \pm 0.009 M_{\odot}$.

From the mass function we deduce a minimum mass of $0.41 M_{\odot}$ for the companion, and, assuming a maximum mass of $1.35 M_{\odot}$ for a white dwarf companion, the inclination is constrained to $31<i<65^{\circ}$. A lower inclination is expected for a more massive neutron star companion. A main-sequence companion of $0.4 M_{\odot}$ with $M_{V} \approx 10$, comparable to LP 400-22, would have left the obvious spectroscopic signatures of a red dwarf near $\mathrm{H} \alpha$, so that candidacy may be rejected.
Table 3. Revised white dwarf parameters.

\begin{tabular}{llc}
\hline \hline Parameter & Measurement & Refs. $^{a}$ \\
\hline$T_{\text {eff }}$ & $11080 \pm 140 \mathrm{~K}$ & 1 \\
& $11290 \pm 50 \mathrm{~K}$ & 2 \\
$\left\langle T_{\text {eff }}\right\rangle$ & $11140 \pm 90 \mathrm{~K}$ & 3 \\
$\log g$ & $11170 \pm 90 \mathrm{~K}$ & 3 \\
& $6.32 \pm 0.08(\mathrm{cgs})$ & 1 \\
& $6.30 \pm 0.02(\mathrm{cgs})$ & 2 \\
$\langle\log g\rangle$ & $6.42 \pm 0.06(\mathrm{cgs})$ & 3 \\
$M^{b}$ & $6.35 \pm 0.05(\mathrm{cgs})$ & 3 \\
Cooling age & $0.190 \pm 0.004 M_{\odot}$ & 3 \\
$M_{V}$ & $1.83 \pm 0.17 \mathrm{Gyr}$ & 3 \\
distance & $4.98 \pm 0.12 \mathrm{mag}$ & 3 \\
$(U, V, W)$ & $(-405 \pm 40 \mathrm{pc}$ & 3 \\
\hline
\end{tabular}

${ }^{a}$ References: (1) Kawka et al. (2006); (2) Kilic et al. (2009); (3) This work. ${ }^{b}$ Mass based on $Z=0.001$ models (Serenelli et al. 2002).

Assuming that the white dwarf is tidally locked to its unseen companion, the expected rotational velocity is $v_{\text {rot }} \approx 2 \mathrm{~km} \mathrm{~s}^{-1}$. This effect is not measurable even when using our best data (KPNO) at a velocity resolution of $37 \mathrm{~km} \mathrm{~s}^{-1}$.

\subsection{Properties of the white dwarf}

Figure 3 compares the observed parameters of LP 400-22 and other ELM white dwarfs with masses below $0.2 M_{\odot}$ to the evolutionary models with solar metallicity progenitors (Serenelli et al. 2001) and with reduced metallicity (Serenelli et al. 2002). The isochrones are labelled with $\log$ (years) and the curves of constant mass are labelled in units of $M_{\odot}$. The effect of an increased metallicity is to reduce the age for a given effective temperature and surface gravity. The age and mass of LP400-22 derived by Kawka et al. (2006) were based on high-metallicity models under the assumption of a relatively short total age. If we accept the possibility that the kinematics of the system is a consequence of old age, then low-metallicity models are appropriate. In this case we re-evaluate the mass at $M=0.19 M_{\odot}$ 


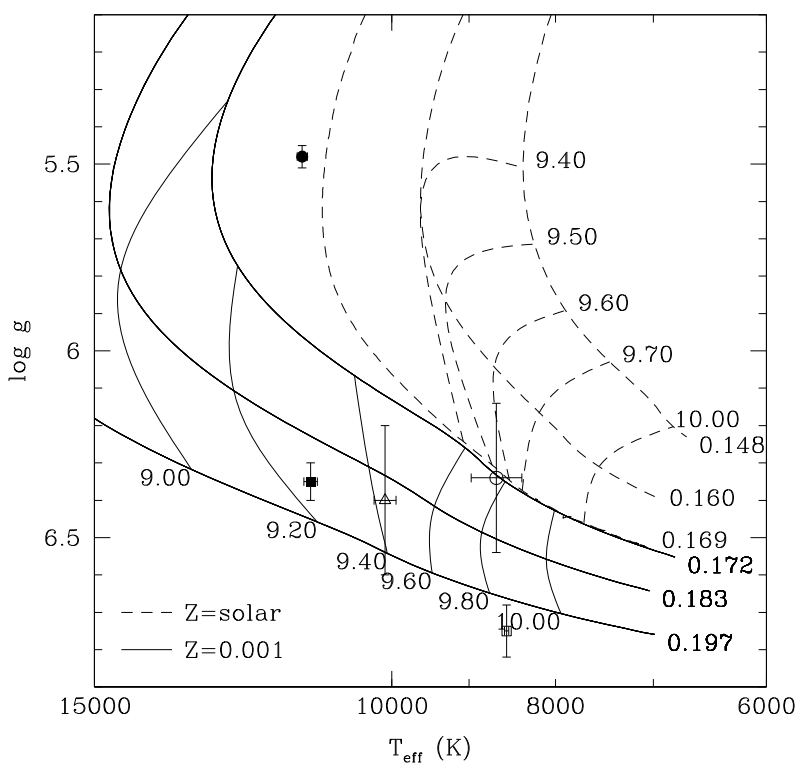

Fig. 3. Physical parameters of ELM white dwarfs compared to evolutionary models. The ELM white dwarfs are LP 400-22 (full square), SDSS J0917+4638 (full circle, Kilic et al. 2007a,b), and the companions to PSR J1911-5958A (open triangle Bassa et al. 2006) and PSR J1012+5307 (open square or open circle, van Kerkwijk et al. 1996; Callanan et al. 1998, respectively). The evolutionary models with solar metallicity are from Serenelli et al. (2001) and the low-metallicity models $(Z=0.001)$ are from Serenelli et al. (2002).

and the age at $t_{\text {cool }}=1.8 \mathrm{Gyr}$. The corresponding absolute magnitude $M_{V}=8.98 \pm 0.12 \mathrm{mag}$, from which we infer a distance modulus $m-M=8.24 \pm 0.14$ and a distance of $444 \mathrm{pc}$. Table 3 presents revised properties of the white dwarf. Assuming a thick disk or halo origin for the system $\left(t_{\text {total }} \approx 8-13 \mathrm{Gyr}\right)$ implies a pre-RLOF duration, taken as the time for exhaustion of core hydrogen, of 6-11 Gyr. Based on evolutionary tracks at $Z=0.001$ of Girardi et al. (2000), the mass of the white dwarf progenitor is constrained to $0.85 \lesssim M \lesssim 1 M_{\odot}$. Interestingly, the case for a low-metallicity progenitor for LP400-22 is supported by the neighbouring case of PSRJ1911-5958A, which is a probable member of the old cluster NGC 6752 (Gratton et al. 2003) characterised by an age of 13.8 Gyr and low metallicity $[\mathrm{Fe} / \mathrm{H}]=-1.43(Z=0.001)$. With an estimated cooling age of $2.5 \mathrm{Gyr}$, a progenitor lifetime of $11.3 \mathrm{Gyr}$ is inferred corresponding to a mass $\sim 0.85 M_{\odot}$.

The white dwarfs depicted in Fig. 3 are old, from $\sim 1$ to $6 \mathrm{Gyr}$, and have masses narrowly distributed around $0.18 M_{\odot}$. Their masses and orbital periods of 14.5 (PSRJ1012+5317), 20.0 (PSRJ1911-5958A), and $24.2 \mathrm{~h}$ (LP400-22) provide an anchor for the final mass-period relations for these systems (Rappaport et al. 1995; Nelson et al. 2004; Benvenuto \& De Vito 2005). Assuming negligible orbital evolution since the last ROLF episode, the three systems yield a final mass and period of $\left(M_{\mathrm{f}} / M_{\odot}, \log P_{\mathrm{f}}(d)\right)=(0.18,-0.1)$. With possibly the lowest mass $\left(\lessgtr 0.17 M_{\odot}\right)$ of the sample and shortest orbital period (7.6 h), the white dwarf SDSS J0917+4638 extends the trend toward shorter periods.

\section{Discussion}

We show that the binary LP400-22 that comprises an ELM white dwarf has a period of $1.01016 \pm 0.00005 \mathrm{~d}$. Consistent with the kinematics, we adopted low metallicity models implying a mass of $0.190 \pm 0.004$ for the white dwarf and a mass ratio $q=M_{\mathrm{wd}} / M_{\mathrm{comp}}<0.477$. The cooling age of the white dwarf is $t_{\text {cool }}=1.8 \pm 0.2 \mathrm{Gyr}$. If adopting a total age for the system of at least $8 \mathrm{Gyr}$, the progenitor of the white dwarf may have been a late $\mathrm{G}$ star with a mass $\lesssim 1 M_{\odot}$.

A possible evolutionary scenario initially involved a $\mathrm{G}+\mathrm{O}$ type binary that subsequently evolved through a $\mathrm{CE}$ phase which preceded a supernova explosion and that was followed by an episode of RLOF from the evolving $\mathrm{G}$ star onto the neutron star (Willems \& Kolb 2002; Benvenuto \& De Vito 2005). In this case, 1.8 Gyr ago during an RLOF episode, LP400-22 would have appeared as a low-mass X-ray binary. Scenarios involving a white dwarf accretor rather than a neutron star are also possible (Nelemans et al. 2001; Nelson et al. 2004). Upon certain assumptions concerning the cooling history, Nelemans et al. (2001) successfully produce ELM white dwarfs in close binaries. An episode of RLOF also implies a past appearance as a cataclysmic variable.

The derived velocity vectors $(U, V, W)$ are not typical of the halo (Kawka et al. 2006; Kilic et al. 2009), and it is possible that the Galactic orbit was altered by a supernova event that added a kick velocity of $\gtrsim 100 \mathrm{~km} \mathrm{~s}^{-1}$ to the system (Hobbs et al. 2005; Kiel \& Hurley 2009). However, it is unlikely that a supernova event would induce such a large total velocity without disrupting the binary in the process. Therefore, the kinematical properties of this relatively nearby system helps establish its halo population membership and old age.

Acknowledgements. S.V. and A.K. are supported by grants IAA300030908 and IAA301630901 from the GA AV, respectively. Based on observations obtained with the Apache Point Observatory 3.5-m telescope, which is owned and operated by the Astrophysical Research Consortium.

\section{References}

Agüeros, M. A., Heinke, C., Camilo, F., et al. 2009, ApJ, 700, L123 Bassa, C. G., van Kerkwijk, M. H., Koester, D., \& Verbunt, F. 2006, A\&A, 456, 295

Benvenuto, O. G., \& De Vito, M. A. 2005, MNRAS, 362, 891

Callanan, P. J., Garnavich, P. M., \& Koester, D. 1998, MNRAS, 298, 207

Chiba, M., \& Beers, T. C. 2000, AJ, 119, 2843

Girardi, L., Bressan, A., Bertelli, G., \& Chiosi, C. 2000, A\&AS, 141, 371

Gratton, R. G., Bragaglia, A., Carretta, E., et al. 2003, A\&A, 408, 529

Hobbs, G., Lorimer, D. R., Lyne, A. G., \& Kramer, M. 2005, MNRAS, 360, 974

Kawka, A., Vennes, S., Oswalt, T. D., Smith, J. A., \& Silvestri, N. M. 2006, ApJ, 643, L123

Kiel, P. D., \& Hurley, J. R. 2009, MNRAS, 395, 2326

Kilic, M., Allende Prietro, C., Brown, W. R., \& Koester, D. 2007a, ApJ, 660, 1451

Kilic, M., Brown, W. R., Allende Prietro, C., Pinsonneault, M. H., \& Kenyon, S. J. 2007b, ApJ, 664, 1088

Kilic, M., Brown, W. R., Allende Prieto, C., et al. 2009, ApJ, 695, L92

Mazeh, T., Latham, D. W., \& Stefanik, R. P. 1996, ApJ, 466, 415

Nelemans, G., Yungelson, L. R., Portegies Zwart, S. F., \& Verbunt, F. 2001, A\&A, 365, 491

Nelson, L. A., Dubeau, E., \& MacCannell, K. A. 2004, ApJ, 616, 1124

Rappaport, S., Podsiadlowski, P., Joss, P. C., Di Stefano, R., \& Han, Z. 1995, MNRAS, 273, 731

Serenelli, A. M., Althaus, L. G., Rohrmann, R. D., \& Benvenuto, O. G. 2001, MNRAS, 325, 607

Serenelli, A. M., Althaus, L. G., Rohrmann, R. D., \& Benvenuto, O. G. 2002, MNRAS, 337, 1091

van Kerkwijk, M. H., Bergeron, P., \& Kulkarni, S. R. 1996, ApJ, 467, L89

Willems, B., \& Kolb, U. 2002, MNRAS, 337, 1004 